\title{
SURF ZONE WAVE HEATING BY ENERGY DISSIPATION OF BREAKING WAVES
}

\author{
Zhangping Wei ${ }^{1}$, Robert A. Dalrymple ${ }^{2}$
}

\begin{abstract}
This study investigates surf zone wave heating due to the dissipation of breaking wave energy by using the Smoothed Particle Hydrodynamics method. We evaluate the surf zone wave heating by examining the increase of internal energy of the system, which is computed based on the conservation of energy. The equivalent temperature profile is calculated based on a simple conversion relationship between energy and temperature. We first examine the surf zone wave heating based on long-crested wave breaking over a planar beach, and we consider spilling breaker and weakly plunging breaker. Numerical results show that breaking of water waves in the surf zone increases the internal energy of water body. Furthermore, the dissipation of incident wave energy is fully converted into the internal energy in a thermally isolated system, confirming the energy conservation of the present numerical approach. It is further found that the long-crested wave breaking generates undertow, which transports the generated wave heating from the surf zone to deep waters. We further carry out numerical experiments to examine surf zone wave heating due to short-crested wave breaking over a beach. The internal energy generation mainly follows the isolated wave breakers, and there is a 3D pattern of wave heating due to the complicated wave breaking process and current system. In general, the magnitude of the generated internal energy or temperature by dissipation of breaking wave energy in the surf zone is relatively small. The present study shows that the generated water temperature is in the order of $10^{-3}$ Kelvin for wave breaking over a typical coastal beach.
\end{abstract}

Keywords: Surf zone, Wave heating; Wave breaking; Energy dissipation; Energy conservation; Smoothed particle hydrodynamics; Wave-driven currents.

\section{INTRODUCTION}

Wave breaking in the surf zone drives complicated turbulent structures (Nadaoka et al., 1989; Lakehal and Liovic, 2011; Wei et al., 2018). It also transfers momentum from waves with relatively high frequencies into low-frequency rotational motions (Clark et al., 2012; Wei et al., 2017). Furthermore, wave breaking induced currents play important roles in nearshore sediment transport (Thornton, 1973; Aagaard et al., 2002). Moreover, wave breaking dissipates the incident wave energy (Rapp and Melville, 1990; Iafrati, 2011). The present work concerns the role of wave breaking in dissipating energy, and particularly the transfer of incident wave energy into wave heating based on the principle of energy conservation in an isolated system.

The variation of water temperature has a direct influence on the development and the substitutability of ecological system in the surf zone, e.g., fish abundance (Harborne and Mumby, 2011). Existing research on water temperature from the inner self to nearshore zone has been carried out by field observation and numerical simulation. For instance, Pineda (1994) reported field measurements of current and temperature at two nearshore stations: Del Mar and Scripps Pier in southern California and found that internal tidal bores play important roles in the water temperature fluctuation. Kumar et al. (2015) compared the field measurement of the water temperature profile in the scale of mid-shelf to surf zone with prediction from a coupled wave and circulation model. In general, previous numerical and field studies reveal that there are temporal variability and vertical structure associated with water temperature in the nearshore zone. However, the fundamental surf zone heat budget, in particular the role of incident wave energy contribution to wave heating, has been less understood. Sinnett and Feddersen (2014) presented a surf zone heat budget based on 47 days field observations at Scripps Pier, and their study showed that viscous and turbulent dissipation of breaking waves contributed to surf zone wave heating. Inspired by the work of Sinnett and Feddersen (2014), this study conducts a numerical experiment based on a phase-resolving wave model to examine wave heating by dissipation of breaking wave energy in the surf zone.

To realistically model surf zone process, the 3D wave breaking (i.e., free surface overturning) needs to be well resolved. The mesh-free Smoothed Particle Hydrodynamics (SPH) method (Gingold and Monaghan, 1977; Lucy, 1977) has gained popularity over mesh-based methods in simulating nonlinear wave dynamics in the past decade (Dalrymple and Rogers, 2006; Makris et al., 2016; Wei et al., 2017). By solving the thermal temperature (or thermal energy) equation, the SPH method can be further applied to heat conduction and diffusion problems, e.g., lava flows (Hérault et al., 2011). It is further feasible to add the viscosity effect into the thermal temperature equation (Cleary and Ha, 2003). However, the thermal energy

\footnotetext{
${ }^{1}$ Department of Civil Engineering, Johns Hopkins University, Baltimore, MD 21218, USA (corresponding author). E-mail: zwei@jhu.edu; zwei.coast@gmail.com

${ }^{2}$ Department of Civil Engineering, Johns Hopkins University, Baltimore, MD 21218, USA. E-mail: rad@jhu.edu
} 
approach does not conserve the energy in the system (Monaghan, 2005). According to the First Law of Thermodynamics, different forms of energy in a system can be transferred between them. Furthermore, considering a thermally isolated system, its internal energy could be directly converted from the work energy done to the system by external environment. Since the primary focus of this work is to evaluate surf zone wave heating converted from the incident wave energy (i.e., the work done by the wavemaker in a wave basin), it is preferable to use the increase of internal energy of the system as an indicator of surf zone wave heating in this work.

This study presents a numerical study of surf zone wave heating generated by energy dissipation of breaking waves using the open-source GPUSPH model. We take advantage of the capability of SPH to resolve breaking waves, and further obtain the internal energy due to dissipation of breaking waves by utilizing the First Law of Thermodynamics. The primary focuses of this work are (1) the energy budget between the incident wave energy and the surf zone internal energy, and (2) the role of wave-driven currents in transporting of wave heating. The rest of the paper is organized as follows. We first introduce the background of the numerical model GPUSPH and our mathematical method to examine surf zone wave heating. We then examine surf zone wave heating over a planar beach generated by long-crested wave breaking. Next, we investigate surf zone wave heating due to short-crested wave breaking over a beach, and the role of rip current in transporting of wave heating. Finally we summarize the findings of this work.

\section{NUMERICAL MODEL}

The numerical model used in this work is based on the weakly compressible Smoothed Particle Hydrodynamics method (Gingold and Monaghan, 1977; Lucy, 1977). Specifically, we use the open-source SPH model GPUSPH to examine surf zone wave heating. The SPH formulation of GPUSPH implemented by Hérault et al. (2010) on graphics processing units (GPUs) can be found, e.g., in Dalrymple and Rogers (2006), as outlined below.

$$
\begin{gathered}
\frac{\mathrm{D} \rho}{\mathrm{D} t}=-\rho \nabla \cdot \mathbf{u} \\
\frac{\mathrm{D} \mathbf{u}}{\mathrm{D} t}=-\frac{\nabla P}{\rho}+\mathbf{g}+v_{0} \nabla^{2} \mathbf{u}+\frac{1}{\rho} \nabla \cdot \tau
\end{gathered}
$$

where $t$ is the time; $\rho$ is the fluid density; $\mathbf{u}$ is the particle velocity; $P$ is the pressure; $\mathbf{g}$ is the gravitational acceleration; $v_{0}$ is the laminar kinematic viscosity; and $\tau$ is the turbulence stress tensor, which is approximated by the sub-particle scale (SPS) model as

$$
\tau_{i, j}=2 \rho v_{t}\left(S_{i, j}-\frac{1}{3} \delta_{i, j} S_{k, k}\right)-\frac{2}{3} \rho C_{I} \Delta^{2} \delta_{i, j}|S|^{2}
$$

where the constant parameter $C_{I}=0.0066 ; \Delta$ is the initial particle spacing; and $\delta_{i, j}$ is the Kronecker delta. The rate of strain tensor is defined by

$$
S_{i, j}=\frac{1}{2}\left(\frac{\partial u_{i}}{\partial x_{j}}+\frac{\partial u_{j}}{\partial x_{i}}\right)
$$

The turbulent viscosity is determined by the Smagorinsky turbulent model:

$$
v_{t}=\left(C_{\text {smag }} \Delta\right)^{2}|S|
$$

where $C_{\text {smag }}=0.12$, and $|S|=\sqrt{2 S_{i, j} S_{i, j}}$.

To close Eqs. (1) and (2), the pressure needs to be determined. The fluid is assumed to be weakly compressible, then the pressure can be directly computed by using the equation of state (Monaghan, 1992) as follows

$$
P=\beta\left[\left(\frac{\rho}{\rho_{0}}\right)^{\gamma}-1\right]
$$

where $\rho_{0}$ is the initial density; $\gamma$ is chosen to be 7 ; and the parameter $\beta$ is calculated by

$$
\beta=\frac{\rho_{0} C_{s}^{2}}{\gamma}
$$


Table 1: The incident wave conditions for two numerical experiments. $h$ : water depth, $H$ : wave height, $T$ : wave period, $L$ : wave length, $k$ : wave number, $\zeta_{0}:$ the surf similarity parameter.

\begin{tabular}{cccccccc}
\hline Index & $\mathrm{h}(\mathrm{m})$ & $\mathrm{H}(\mathrm{m})$ & $\mathrm{T}(\mathrm{s})$ & $\mathrm{L}(\mathrm{m})$ & $\mathrm{kh}$ & $\zeta_{0}$ & \\
\hline 1 & 0.484 & 0.176 & 1.0 & 1.51 & 2.02 & 0.29 & spilling breaker \\
\hline 2 & 0.484 & 0.138 & 1.7 & 3.30 & 0.93 & 0.54 & weakly plunging breaker \\
\hline
\end{tabular}

where $C_{s}$ is the speed of sound. The real speed of sound leads to a very small time step, which is not practical for numerical simulation. Monaghan (1994) suggested to limit the density fluctuation $<1 \%$ by setting the ratio of $C_{s} / u_{\max } \geq 10$. The maximum velocity $u_{\max }$ in this work is defined as $u_{\max }=\sqrt{2 g h}$ (where $h$ is the still water depth).

GPUSPH has been successfully used to investigate a series of topics in the fields of coastal and hydraulic engineering, such as tsunami-structure interaction (Wei et al., 2015; Wei and Dalrymple, 2016; Wei et al., 2016), two-phase sediment laden flows (Shi et al., 2017), and vorticity generation and 3D nearshore circulation under short-crested wave breaking (Wei et al., 2017; Wei and Dalrymple, 2017a,b).

This study evaluates the surf zone wave heating by examining the increase of the internal energy of the system due to the incident wave field. The First Law of Thermodynamics reads

$$
\Delta U=Q-W
$$

This law states that the change in the internal energy $(\Delta U)$ of a closed system is equal to the amount of heat $(Q)$ supplied to the system, minus the amount of work $(W)$ done by the system on its surroundings. The numerical wave basin considered in this study generates wave offshore, and then the generated waves propagate and eventually break over a beach. The wave basin is thus considered to be a thermally isolated system, which does not exchange mass flow and heat energy. As a result, the change of internal energy is solely due to the exchange of the work energy. In other words, the increase of internal energy in the wave basin is solely due to the incident wave energy offshore. This relationship provides us a simple and straightforward approach to examine surf zone wave heating.

Calculation of internal energy $(U)$ in GPUSPH follows the work of Fulk (1994),

$$
\frac{\mathrm{D} U}{\mathrm{D} t}=-\frac{1}{2} m \frac{\mathrm{Du}}{\mathrm{D} t} \cdot \mathbf{u}
$$

where $m$ is the particle mass; $\frac{\mathrm{Du}}{\mathrm{D} t}$ has been defined in Eq. (2), which is the equation of motion of the SPH particle. By using Eq. (2) we make sure that all work energy on the particle is converted to the particle's internal energy.

\section{SURF ZONE WAVE HEATING UNDER LONG-CRESTED WAVE BREAKING}

In this section we examine surf zone wave heating by energy dissipation of long-crested wave breaking over a planar beach. We first introduce the numerical experiment setup, and then we examine the internal energy and temperature increase in the surf zone, and further verify the energy budget in the system.

\section{Numerical experiments}

We present two numerical experiments on monochromatic wave breaking over a planar beach, and the wave parameters are listed in Table 1 . The offshore water depth $(h)$ is about $0.484 \mathrm{~m}$ for both cases, the generated wave heights $(H)$ are $0.176 \mathrm{~m}$ and $0.138 \mathrm{~m}$, respectively. The wave periods $(T)$ are $1.0 \mathrm{~s}$ and $1.7 \mathrm{~s}$, and by using the linear dispersion relationship, we obtain the corresponding wave lengths $(L) 1.51 \mathrm{~m}$ and $3.30 \mathrm{~m}$, respectively. The numerical wavemaker is placed at about one wave length away from the planar beach toe. The wave depth parameter $k h$ (where $k$ is the wave number) shows that both waves are intermediate-depth waves. Finally, we estimate the breaker type based on the surf similarity parameter of Battjes (1975),

$$
\zeta_{0}=\frac{\tan \alpha}{\sqrt{H_{0} / L_{0}}}
$$

where $H_{0}$ is the deep water wave height; $L_{0}$ is the deep water wave length; $\alpha$ is the beach slope, and $\alpha=$ 0.1 is used in this work. The obtained $\zeta_{0}$ in Table 1 shows that the first case generates a spilling breaker, 


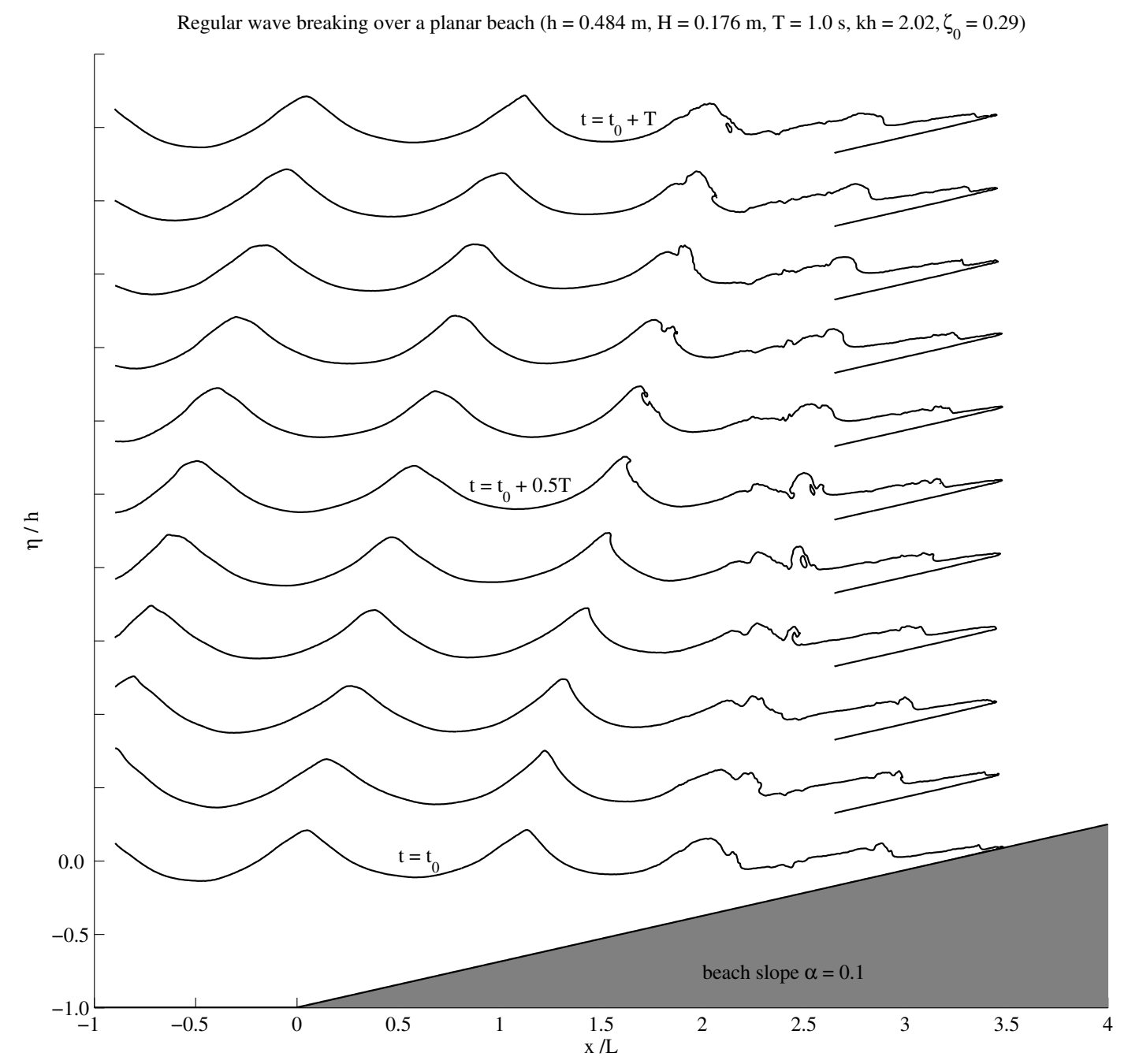

Figure 1: The development of a spilling breaker over a planar beach over one wave period from $t=t_{0}$ to $t$ $=t_{0}+T$. Each line indicates the wave profile at different time levels, and the time increases from the line in the bottom to the top with an interval of $0.1 T$.

and the other case generates a weakly plunging breaker. The numerical discretization utilizes a constant SPH particle size $\Delta p=0.005 \mathrm{~m}$, resulting in about 97 particles to represent the water depth, and about 28 particles to represent the smaller wave height $H=0.138 \mathrm{~m}$.

The breaking wave profile over one wave period from $t=t_{0}$ to $t=t_{0}+T$ for the spilling breaker case is shown in Fig. 1. Each line indicates the breaking wave profile at different time levels, and the time interval is $0.1 T$ between them. As the wave propagates over the planar beach, it is subject to wave shoaling, resulting in the steady increase of wave height from $t=t_{0}$ to $t=t_{0}+0.4 T$. Eventually, the wave front becomes unstable at $t=t_{0}+0.5 T$ and collapses at $t=t_{0}+0.6 T$, generating turbulent water spilling down the face of the wave at $t=t_{0}+0.7 T$. The turbulent bore continues to propagate toward the shore. We can observe from Fig. 1 that the breaking point (or the starting point of the surf zone) is around $x / L=1.25$ for the spilling breaker case.

The breaking wave profile over one wave period from $t=t_{0}$ to $t=t_{0}+T$ for the weakly plunging breaker case is shown in Fig. 2. As the wave propagates over the planar beach, it is also subject to wave shoaling, resulting in a nearly vertical wave front at $t=t_{0}+0.2 T$. The wave front continues to grow and form a curved tip at $t=t_{0}+0.3 T$. The plunging jet is developed at $t=t_{0}+0.4 T$, and it penetrates into the wave front and generates an air packet at $t=t_{0}+0.5 T$. The breaking wave front collapses and generates 


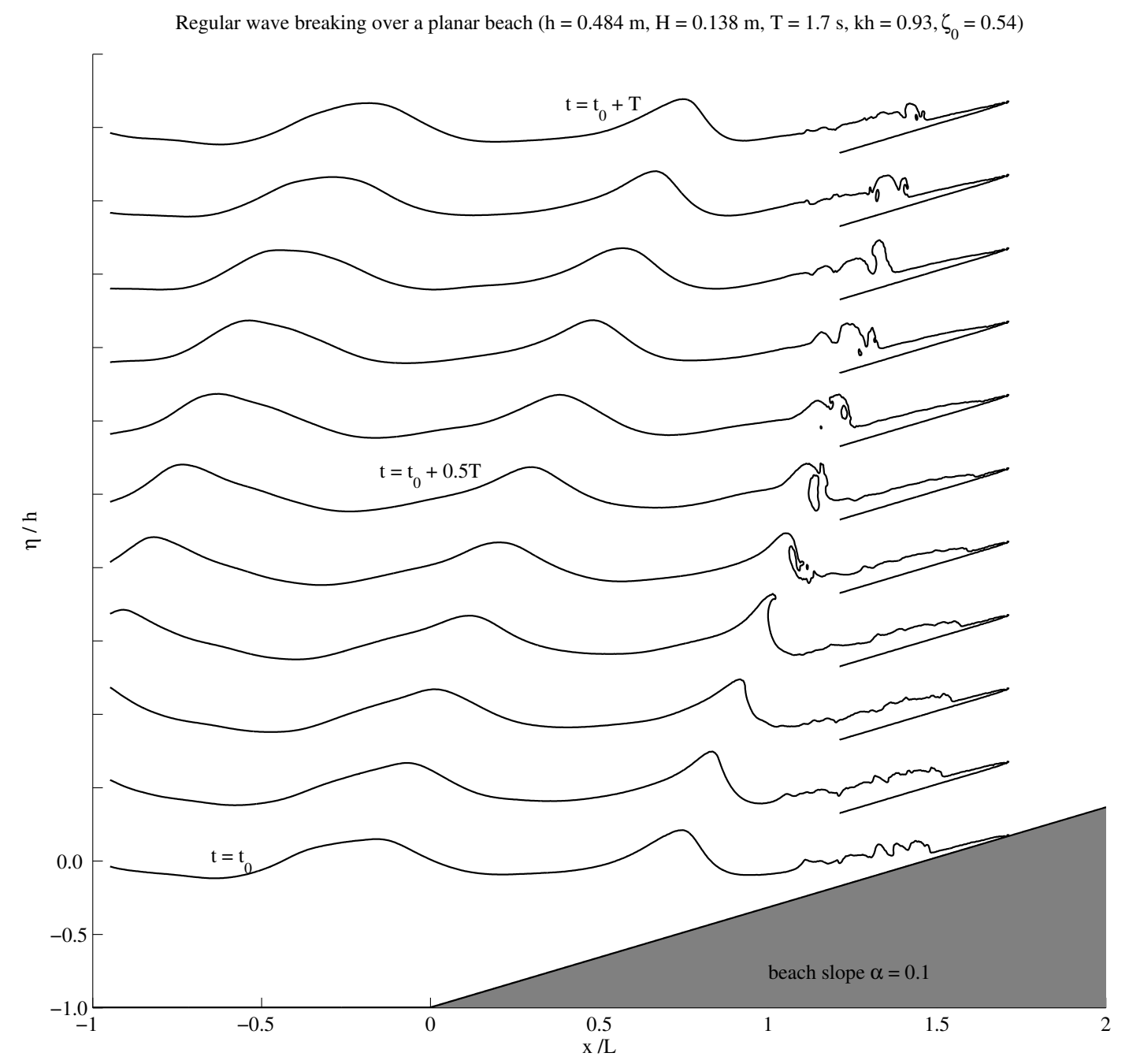

Figure 2: The development of a plunging breaker over a planar beach over one wave period from $t_{0}$ to $t=$ $t_{0}+T$. Each line indicates the wave profile at different time levels, and the time increases from the line in the bottom to the top with an interval of $0.1 T$.

turbulent bores at $t=t_{0}+0.7 T$. The turbulent bore continues to propagate toward the shore. A secondary reverse breaker is observed from $t=t_{0}+0.8 T$ to $t=t_{0}+0.9 T$. Fig. 2 shows that the breaking point (or the starting point of the surf zone) starts around $x / L=0.8$ for the weakly plunging breaker case (Note that the weakly plunging breaker case has a longer wave length than that of the spilling breaker case).

\section{Surf zone wave heating: internal energy \& temperature}

The breaking wave profiles in Figs. 1 and 2 show that the wave propagates from offshore and eventually breaks over the planar beach. This section shows the generated wave heating by the dissipation of wave breaking in the surf zone.

Fig. 3 shows the internal energy profile for the spilling breaker case at two instants (a) $t=20 \mathrm{~s} \mathrm{(20T)}$ and (b) $t=30 \mathrm{~s}(30 T)$. We have several important observations: (1) due to the considered relatively small scale wave breaking process, the generated internal energy is very small $\left(\times 10^{-4} \mathrm{~J}\right) ;(2)$ there is no internal energy distributed in the wave propagation region, and the internal energy is mainly generated in the surf zone; (3) the comparison of the internal energy profile at $20 \mathrm{~T}$ in Fig. 3(a) and $30 \mathrm{~T}$ in Fig. 3(b) shows that the internal energy increase over time.

The internal energy profile generated by the weakly plunging breaking waves is shown in Fig. 4. In general, our findings related to internal energy profile under the spilling breaker case are applicable to the 

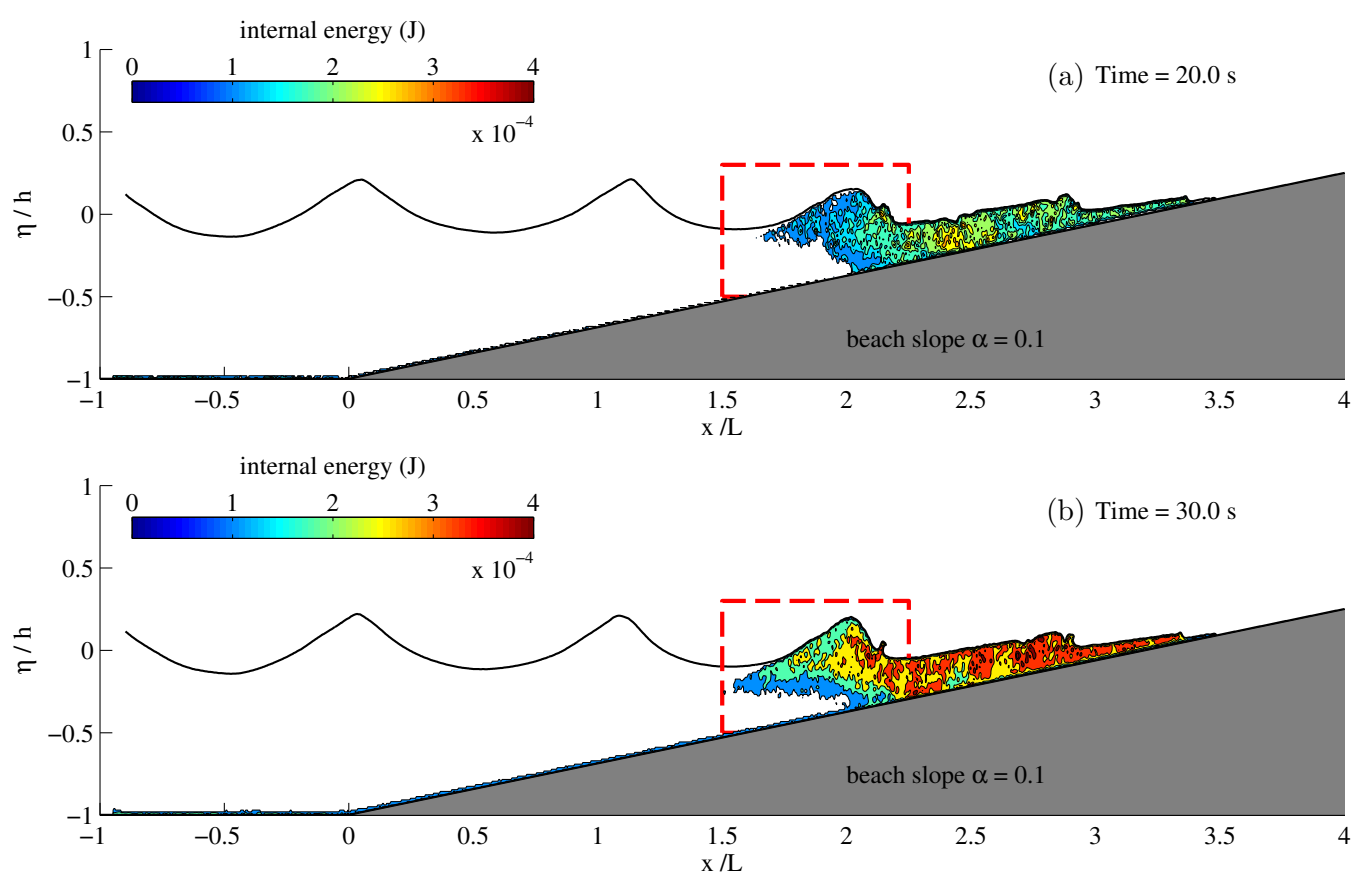

Figure 3: The instantaneous view of internal energy profile for the spilling breaker case at (a) $t=20 \mathrm{~s}(20 \mathrm{~T})$ and (b) $t=30 \mathrm{~s}(\mathbf{3 0} T)$. The two red dashed-line boxes compare the offshore transport of wave heating by the undertow.
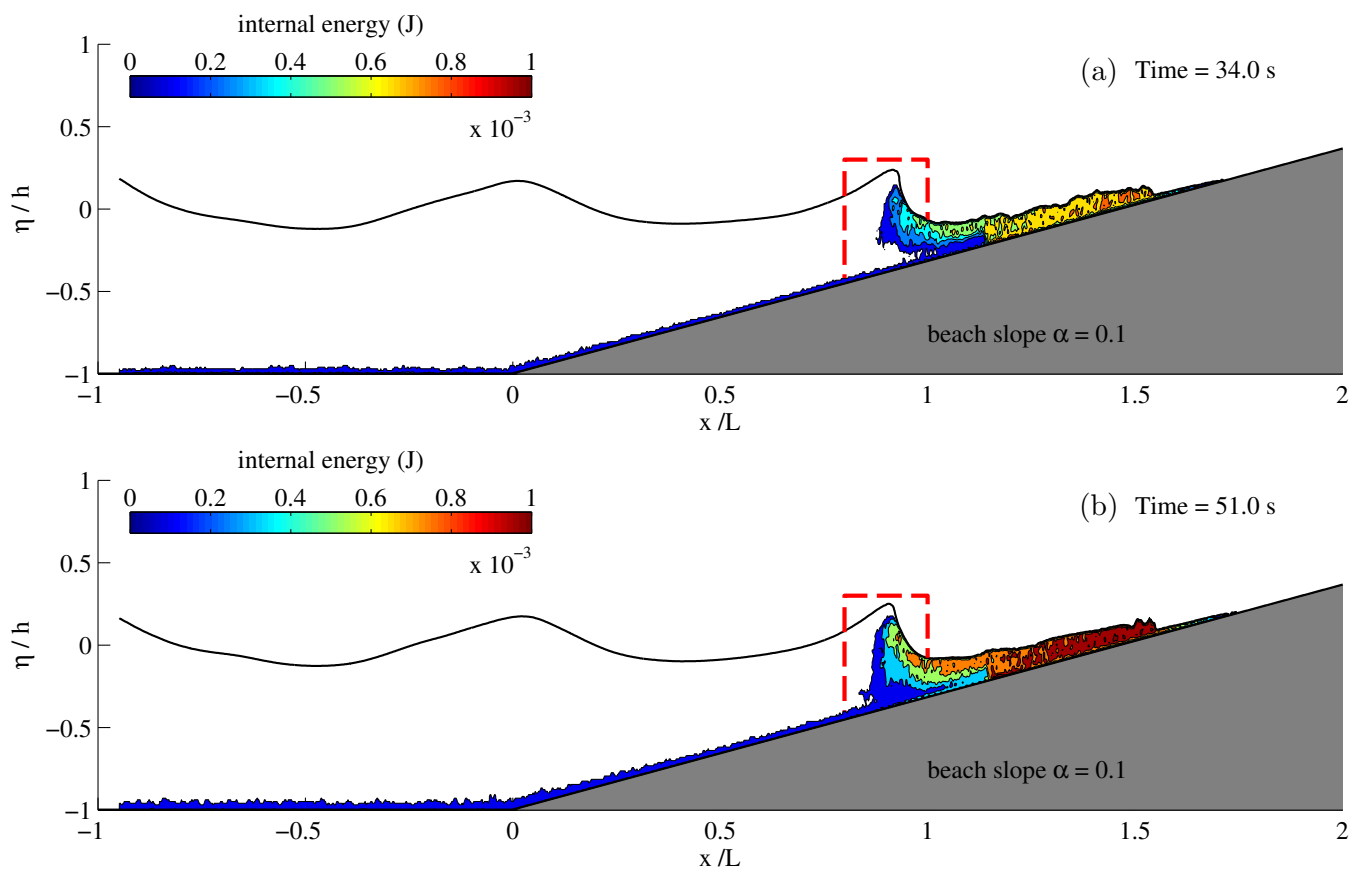

Figure 4: The instantaneous view of internal energy profile for the plunging breaker case at (a) $t=34 \mathbf{s}$ $(20 T)$ and (b) $t=51 \mathbf{s}(30 T)$. The two red dashed-line boxes compare the offshore transport of wave heating by the undertow. 


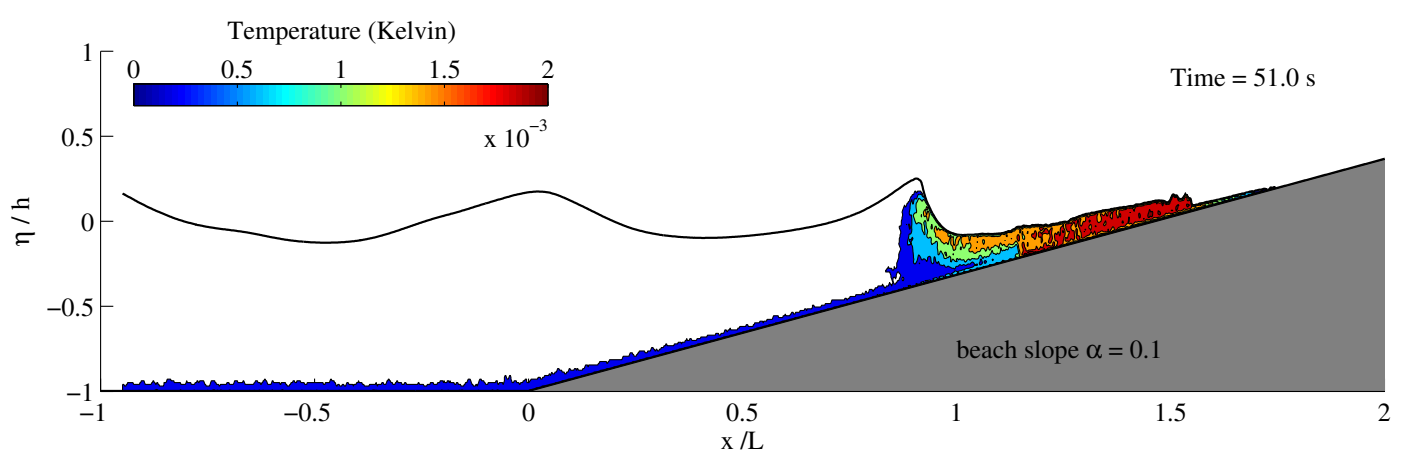

Figure 5: The instantaneous view of temperature profile by conversion from internal energy with Eq. (11) for the plunging breaker case at $t=51 \mathrm{~s}(30 T)$.

weakly plunging breaker case. An additional observation is that the beach is also heated in the system (This is also observed in the spilling breaker case, although it is even weaker). This phenomenon is due to the fact that: (1) the present study considers intermediate-depth waves, as a result, the wave propagation is influenced by the bottom, and (2) the numerical beach in this work is dissipative with zero velocity at the wall boundary.

The surf zone wave heating can be also evaluated by examining the "equivalent" temperature field. We can convert the internal energy into temperature $\left(T_{p}\right)$ by the following relationship,

$$
T_{p}=\frac{U}{c_{p} \cdot m}
$$

where the particle internal energy $U$ has the unit of Joule (or $\frac{K g \cdot \mathrm{m}^{2}}{\mathrm{~s}^{2}}$ ), and $c_{p}$ is the specific heat capacity (unit: $\mathrm{J} /(\mathrm{Kg}$ Kelvin)). Fig. 5 shows the temperature profile for the weakly plunging breaker case at $t=51 \mathrm{~s}$ (30T), and its corresponding internal energy profile was shown in Fig. 4(b). It is seen that the distribution of the temperature profile is very similar to the distribution of its internal energy profile. Furthermore, the generated temperature is also very small for this specific setup, and it is in the order of $10^{-3}$ Kelvin.

\section{Wave heating transport by undertows}

It is well-known that wave breaking in the nearshore zone also drives currents. Considering the longcrested wave breaking presented above, we would expect the development of undertow, an offshore-directed current, below the wave trough. Fig. 6 shows the time-averaged current fields for both breaking wave cases presented above. Ten waves from $t=20 T$ to $30 T$ were averaged. The three red lines (from top to down) indicates the wave crest profile, the mean water level (or the wave set-up and set-down line), and the wave trough profile. The approximate breaking points of waves observed in Figs. 1 and 2 are also indicated in Fig. 6. It is seen that the mean current profile near the surf zone is relatively random compared with the offshore wave propagation region, and indeed undertow is developed under the wave trough in both cases.

To examine the transport of generated wave heating by undertow, we will revisit Figs. 3 and 4, in particular, the internal energy profile highlighted in the red dashed-line boxes. The basic idea is to compare the spatial distribution of internal energy at the two time instants, and examine if there is an increase (in terms of distribution area) of internal energy at $30 T$ than that at $20 T$. It should be pointed out that the wave breaking process is relatively stable after $20 T$, indicating that the location of wave heating generation should be similar at both time instants. By examining the internal energy profiles highlighted in red boxes in Figs. 3 and 4, we can see that the area of internal energy profile at $30 T$ is indeed larger than the area of internal energy profile at 20T. Considering the fact that the undertow transports mass steadily offshore from the surf zone, the increase of internal energy in the outer surf zone over time is attributed to the transport of generated heat from the inner surf zone where the wave heating is generated. In other words, undertow is the carrier of wave heating to the offshore area or deep waters.

\section{Energy budget}

As pointed out earlier, the numerical basin is considered as a "thermally isolated system", which does not exchange mass flow and heat energy with external environment. As a result, the increase of internal 
(a) Mean current field under regular wave breaking over a planar beach $\left(\mathrm{h}=0.484 \mathrm{~m}, \mathrm{H}=0.176 \mathrm{~m}, \mathrm{~T}=1.0 \mathrm{~s}, \mathrm{kh}=2.02, \zeta_{0}=0.29\right)$.

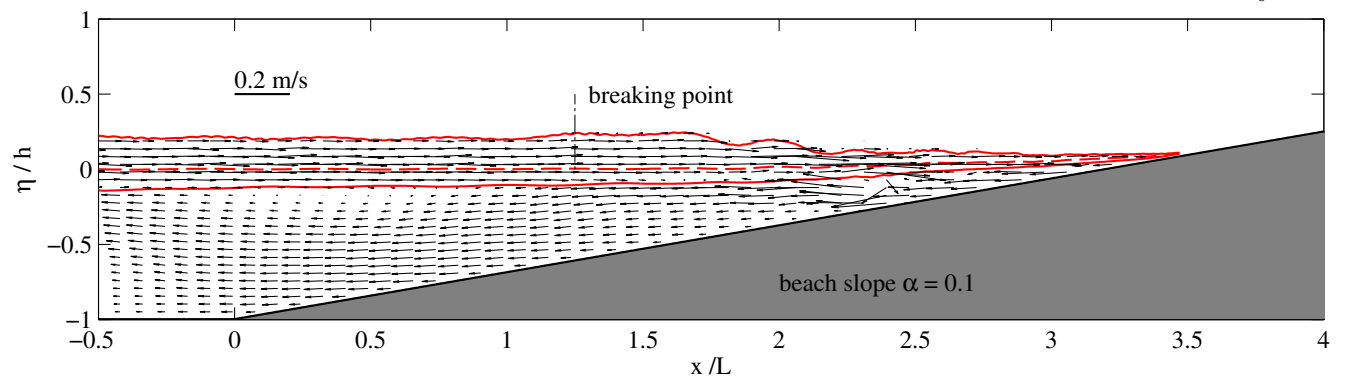

(b) Mean current field under regular wave breaking over a planar beach $\left(\mathrm{h}=0.484 \mathrm{~m}, \mathrm{H}=0.138 \mathrm{~m}, \mathrm{~T}=1.7 \mathrm{~s}, \mathrm{kh}=0.93, \zeta_{0}=0.54\right)$.

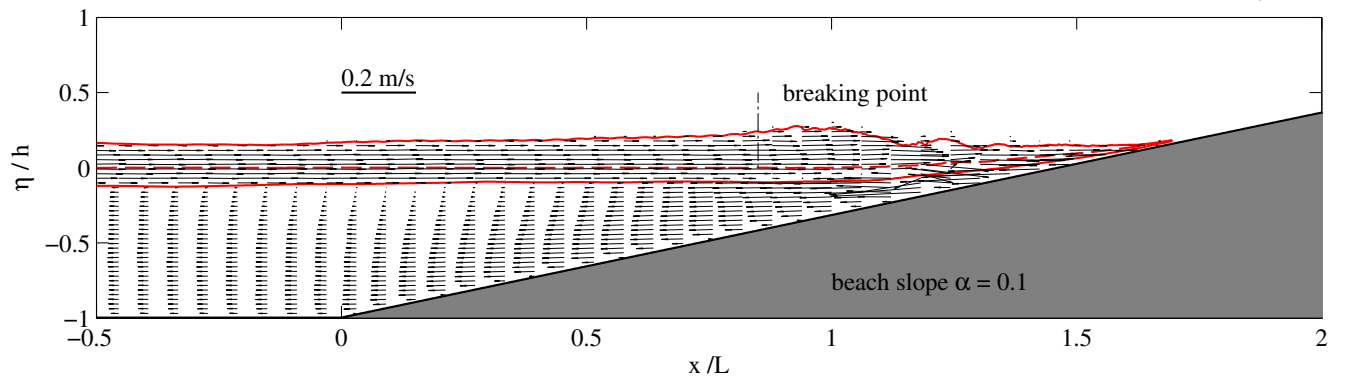

Figure 6: The mean current field by ten-waves averaging from $20 T$ to $30 T$ for (a) the spilling breaker case and (b) the plunging breaker case. Three red lines indicate the wave crest profile, the mean water level, and the wave trough profile, respectively. Note that different velocity vector scales are used in the two subplots.

energy in the system should be equal to the work done by the wavemaker (or the incident wave energy). This section verifies the energy budget in the isolated system by comparing the incident wave energy rate and the internal energy increase rate.

The average incident wave energy per unit surface area $(E)$ is defined as (e.g., Dean and Dalrymple, 1991),

$$
E=\frac{1}{8} \rho_{w} g H^{2}
$$

where $H$ is the wave height; $\rho_{w}$ is the water density; $g$ is the gravitational acceleration. The rate at which the energy is transferred is called the energy flux. The mean energy flux is obtained by averaging the rate at which work is being done by the dynamic pressure over one wave period. As a result, the averaging energy flux per unit width $\left(F_{w}\right)$,

$$
F_{w}=E C_{g}=\frac{1}{8} \rho_{w} g H^{2} C_{g}
$$

where the group velocity $C_{g}$ is determined by

$$
C_{g}=\frac{1}{2}\left(1+\frac{2 k h}{\sinh 2 k h}\right) C
$$

and the wave celerity $(C=L / T)$, with the wave length $(L)$ determined by the linear dispersion relationship:

$$
L=\frac{g}{2 \pi} T^{2} \tanh \frac{2 \pi h}{L}
$$

where $h$ is the local water depth; $k$ is the wave number $\left(k=\frac{2 \pi}{L}\right)$; and $T$ is the incident wave period.

In the SPH simulation, each SPH particle carries scalar properties including the internal energy. Therefore, to compute the internal energy increase rate, we first sum up the total amount of internal energy in the wave basin $\left(U_{s}\right)$, i.e., the summation of the internal energy of each fluid particle, as follows,

$$
U_{s}=\sum_{i=1}^{N_{p}} U_{i}
$$


(a) $\mathrm{h}=0.484 \mathrm{~m}, \mathrm{H}=0.176 \mathrm{~m}, \mathrm{~T}=1.0 \mathrm{~s}$

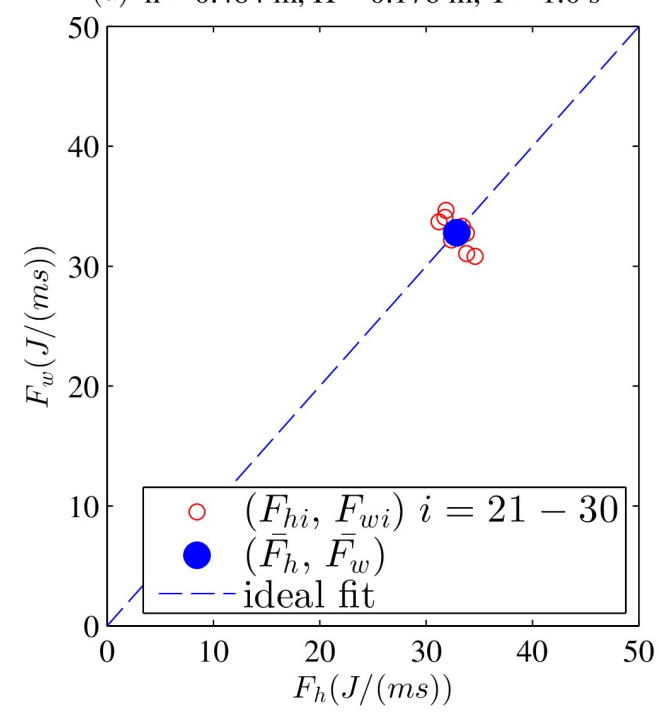

(b) $\mathrm{h}=0.484 \mathrm{~m}, \mathrm{H}=0.138 \mathrm{~m}, \mathrm{~T}=1.7 \mathrm{~s}$

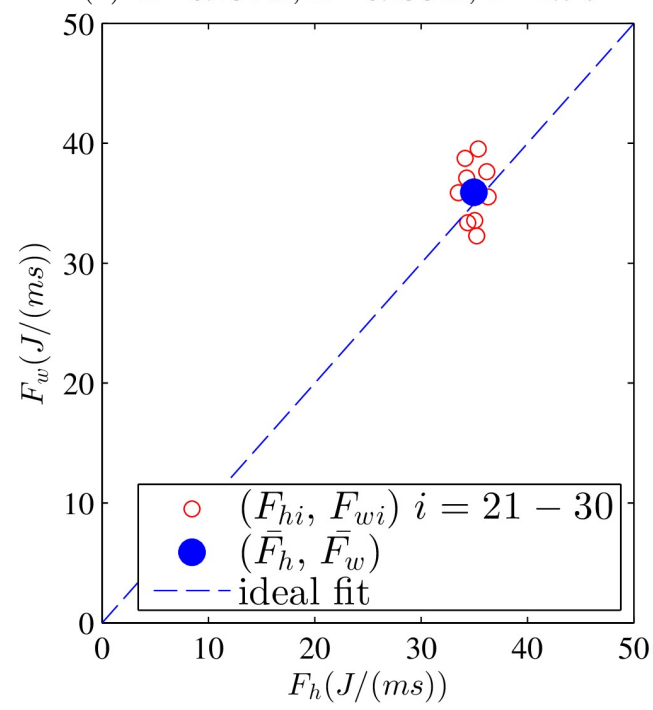

Figure 7: The energy budget estimation based on ten-waves averaging from 20T to 30T for (a) the spilling breaker case and (b) the plunging breaker case.

where $i$ is an index number; and $N_{p}$ is the total number of fluid particles in the wave basin. Then we obtain the averaged rate at which internal energy is generated per unit width $\left(F_{h}\right)$ by

$$
F_{h}=\frac{1}{L_{y}} \frac{\mathrm{D} U_{s}}{\mathrm{D} t}
$$

where $L_{y}$ is the basin width.

To examine the energy conservation in the wave basin, we compare the incident wave energy rate per unit width $\left(F_{w}\right.$ in Eq. (13)) and the internal energy increase rate per unit width $\left(F_{h}\right.$ in Eq. (17)) in Fig. 7. The calculation is based on relatively stable surf zone process from $t=20 T$ to $30 T$. The blue dashed line indicates that the incident wave energy is perfectly converted into the internal energy of the system. We first check the relationship between $F_{w}$ and $F_{h}$ for each wave, and we obtain individual circles as shown in Fig. 7. It is seen that the red circles are scattered around the blue ideal fitting line in both plots. We further compute the ten-waves averaged $F_{w}$ and $F_{h}$, and the result is indicated as blue dots in Fig. 7. We can see that blue dots in both subplots lie closely to the ideal fitting line, confirming that the energy budget is balanced in the present numerical setup.

\section{SURF ZONE WAVE HEATING UNDER SHORT-CRESTED WAVE BREAKING}

In our previous work in Wei et al. (2017), we have presented a simplified representation of short-crested waves by using the intersecting wave train method of Dalrymple (1975). We examined short-crested wave breaking over a planar beach, and observed several important phenomena including isolated breakers, wave amplitude diffraction, wave-current interaction, and vorticity generation. The present study would like to examine the corresponding surf zone wave heating.

\section{Numerical experiments}

The numerical basin setup is briefly reviewed in this section, and the details could be found in Wei et al. (2017). The wave basin is made of an offshore horizontal flat of $b_{x}=4 \mathrm{~m}$ and a planar beach with a mild slope of $m=1: 50$. A series of flap-type wavemakers are located along the $y$ axis at $x=1 \mathrm{~m}$, and the offshore water depth is set to be $h=0.5 \mathrm{~m}$. We consider two incident wave trains with the same wave period of $T=2 \mathrm{~s}$ and the same wave height of $H=0.3 \mathrm{~m}$. The two incident wave angles are equal but opposite $\left(\alpha_{w}=-\beta_{w}=11.71^{\circ}\right)$. We obtain the nodal line spacing $\lambda=10 \mathrm{~m}$ (Dalrymple, 1975). Then the alongshore basin width is chosen to be $L_{y}=2 \lambda=20 \mathrm{~m}$, and two nodal lines are formed at $y=5$ and $15 \mathrm{~m}$ 
alongshore. In GPUSPH, the full wave basin is discretized into particles, with a fixed particle size of $\Delta p=$ $0.02 \mathrm{~m}$, resulting in 25 particles over the water column offshore and a total number of 18.5 million particles for the whole basin. At the two alongshore boundaries, the no-flow wall boundary condition is applied.

\section{Internal energy generation due to short-crested wave breaking}

As mentioned above, to ensure energy conservation the surf zone wave heating is evaluated by examining the internal energy of the system. Fig. 8 shows the top view of internal energy profile at the free surface as the short-crested wave breaks over the planar beach. Two time instants are presented. The first time instant is at $t=56 \mathrm{~s}$ (or 28 waves), and the other time instant is at $t=70 \mathrm{~s}$ (or 35 waves). We have several important observations regarding internal energy generation and distribution: (1) the internal energy is generated in the breaking region; (2) a relatively high internal energy is observed along the trajectories of isolated breakers; (3) there is no or very fewer internal energy generation in the rip channels (i.e., $y=5$ and $15 \mathrm{~m}$ ), and (4) the comparison between two plots shows that the internal energy increases over time.

It is worth pointing out that the internal energy is also transported by wave-driven currents. For the present numerical experiment, rip currents are generated along $y=5$ and $15 \mathrm{~m}$. Fig. 9 (i.e., Figure 8(b) of Wei et al. (2017)) shows the time- and depth-averaged current field colored by the time- and depth-averaged vertical vorticity field generated by short-crested wave breaking along the rip channel at $y=5 \mathrm{~m}$, where the rip currents are located between $x=3 \mathrm{~m}$ and $10 \mathrm{~m}$ and $y=4 \mathrm{~m}$ and $6 \mathrm{~m}$. By comparing the corresponding internal energy profile in Fig. 8(a) and Fig. 8(b), we can see that the internal energy is transported offshore near the rip channel by the rip currents.

\section{D structure of surf zone wave heating}

The above section has shown that wave heating has been generated by the energy dissipation of shortcrested wave breaking in the surf zone. It should be pointed out that there is a 3D nonuniform distribution (in both the horizontal direction and the vertical direction) of short-crested wave breaking in the numerical basin, and we would expect that there should be a 3D distribution of the surf zone wave heating in the basin. Fig. 10 shows the cross-section view of wave heating generation under short-crested wave breaking at $t=$ $70 \mathrm{~s}$ along three sections: $y=10 \mathrm{~m}$ (anti-nodal line), $y=8 \mathrm{~m}$, and $y=5 \mathrm{~m}$ (nodal line or rip channel). The wave heating was expressed as the temperature increase by conversion from internal energy by using Eq. (11). The free surface view of internal energy profile at the three sections was presented in Fig. 8. There is energetic wave breaking along $y=10 \mathrm{~m}$, and we can see that temperature generation in the upper water column in Fig. 10(a). There is alongshore propagation of isolated breakers at cross-section $y=8 \mathrm{~m}$. As a result, we would expect that temperature is generated near isolated breakers (Fig. 10(b)) and there is also alongshore propagation/transport of wave heating cross this section. Since there is no wave breaking in the rip channel at $y=5 \mathrm{~m}$, Fig. 10(c) also confirms that there is almost no wave heating generation along this section. In addition to the 3D structure of temperature profile, Fig. 10 also indicates that there is a beach heating phenomenon caused by a dissipative numerical boundary condition (i.e., zero velocity at the wall boundary) used in the present study. Furthermore, the magnitude of temperature due to wave heating is in the order of $10^{-3}$ Kelvin.

\section{CONCLUSIONS}

We have investigated surf zone wave heating due to the dissipation of breaking wave energy by using the open-source GPUSPH model. By utilizing the First Law of Thermodynamics, we computed the internal energy of the system converted from the work energy done to the system. We evaluated wave heating by examining the increase of internal energy in the surf zone. The equivalent temperature profile was further obtained based on a simple conversion relationship between energy and temperature. Our study addressed surf zone wave heating under different scenarios, such as spilling and plunging breakers, long-crested and short-crested waves. Our major findings are summarized as follows:

- Breaking of water waves in the surf zone increases the internal energy of water body.

- The dissipation of incident wave energy is fully converted into the internal energy in a thermally isolated system as used in the present study.

- Wave-driven currents (undertows and rips) play important roles in transporting the generated internal energy from the surf zone to deep waters (or offshore area). 
(a) Increase in internal energy under short-crested wave breaking $(\mathrm{h}=0.5 \mathrm{~m}, \mathrm{H}=0.3 \mathrm{~m}, \mathrm{~T}=2 \mathrm{~s})$. Time $=56.0 \mathrm{~s}$

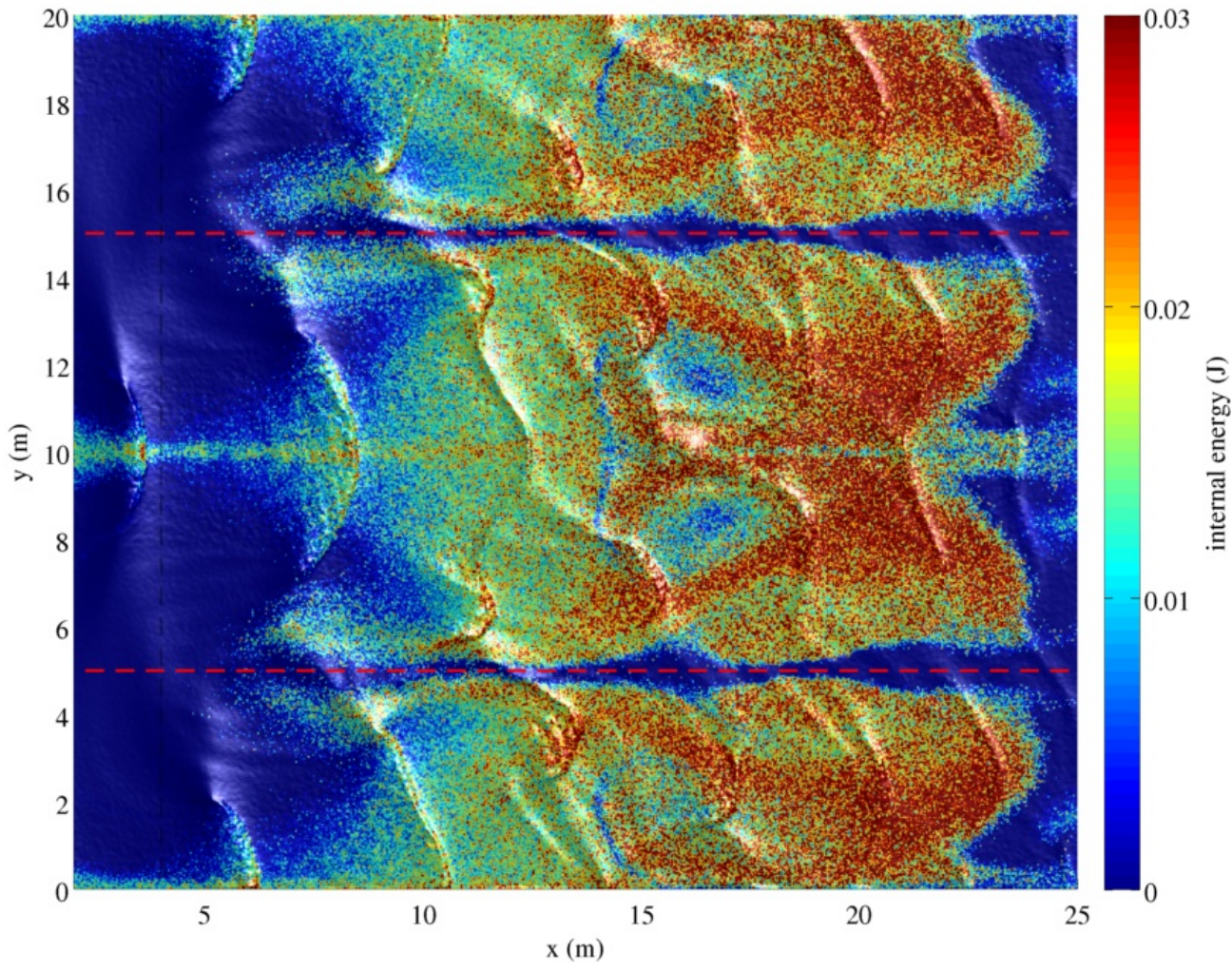

(b) Increase in internal energy under short-crested wave breaking $(\mathrm{h}=0.5 \mathrm{~m}, \mathrm{H}=0.3 \mathrm{~m}, \mathrm{~T}=2 \mathrm{~s})$. Time $=70.0 \mathrm{~s}$

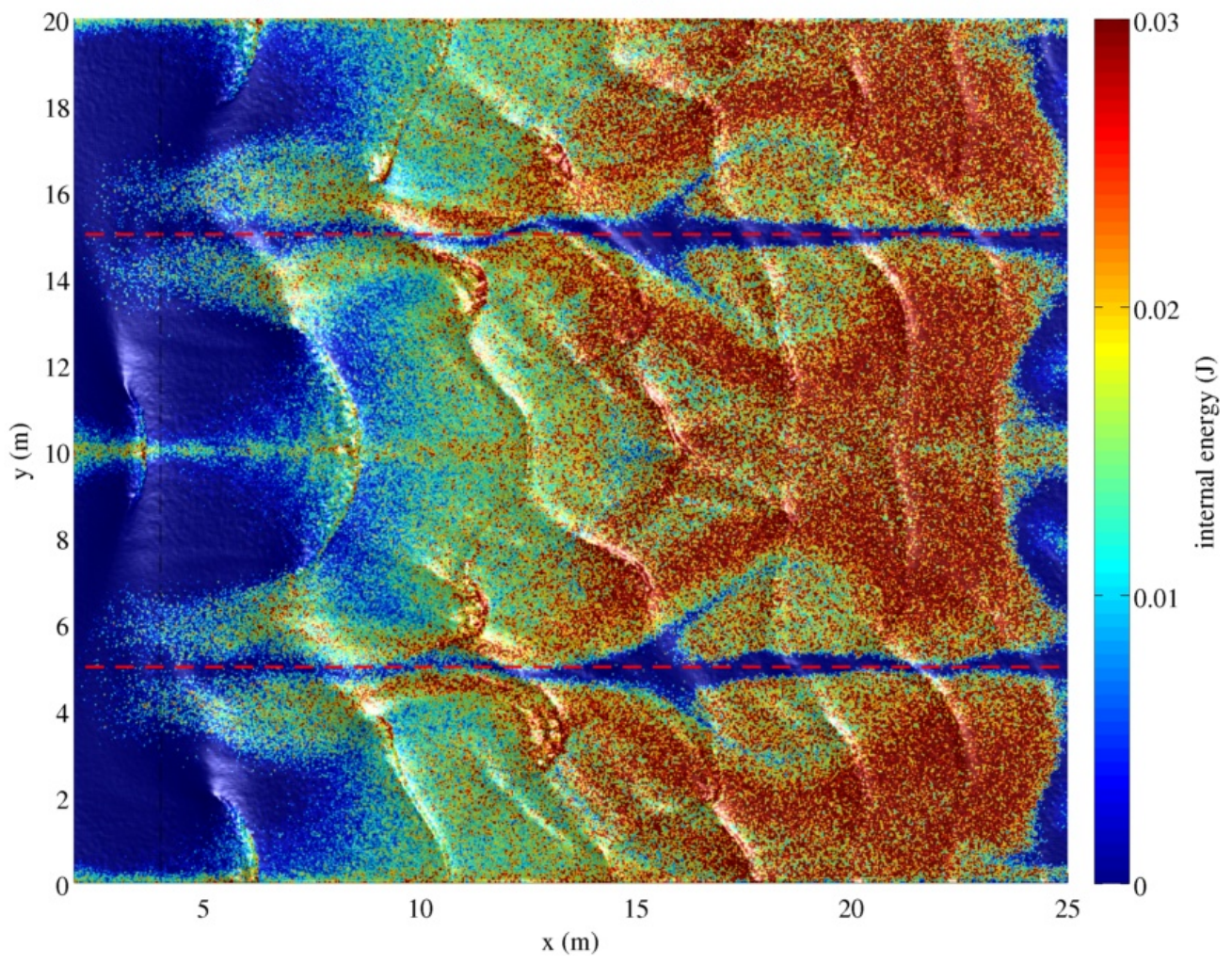

Figure 8: The instantaneous view of internal energy generation by short-crested wave breaking at (a) $t=$ $56 \mathrm{~s}$ and (b) $t=70 \mathrm{~s}$. 


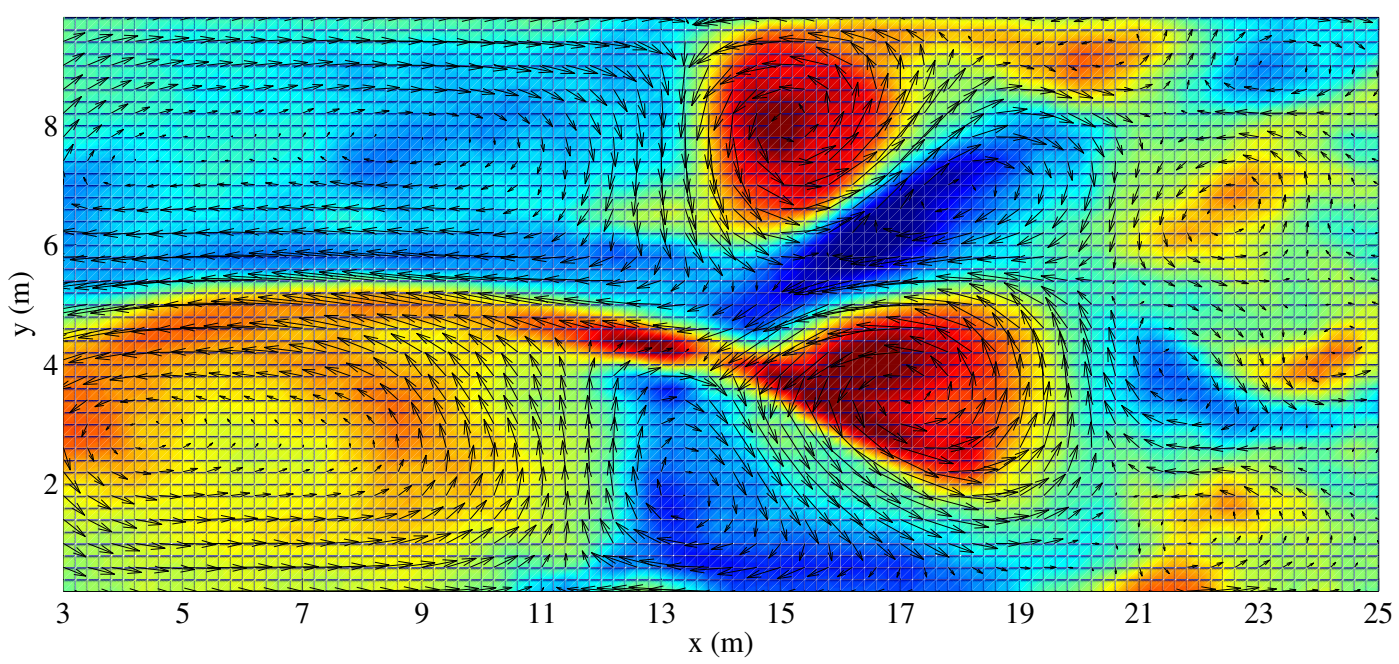

Figure 9: The time- and depth-averaged current field colored by the time- and depth-averaged vertical vorticity field generated by short-crested wave breaking (Figure 8(b) of Wei et al. (2017)).

(a) Increase in temperature under breaking waves at section $\mathrm{y}=10.0 \mathrm{~m}$, time $=70.00 \mathrm{~s}$

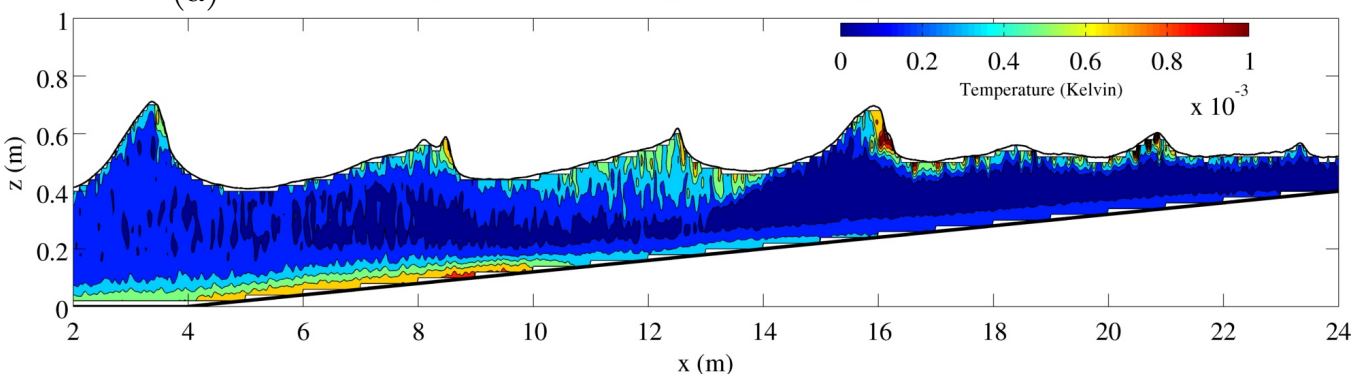

(b) Increase in temperature under breaking waves at section $\mathrm{y}=8.0 \mathrm{~m}$, time $=70.00 \mathrm{~s}$

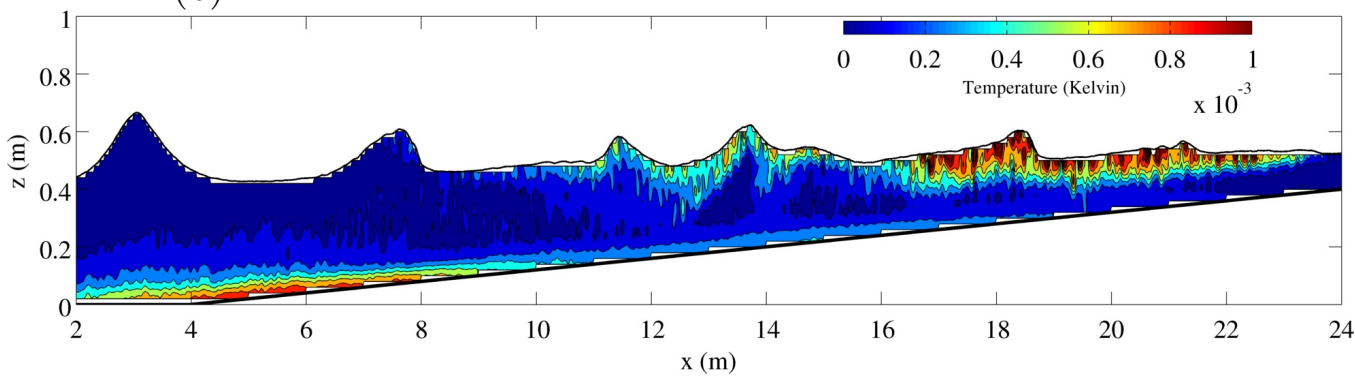

(c) Increase in temperature under breaking waves at section $\mathrm{y}=5.0 \mathrm{~m}$, time $=70.00 \mathrm{~s}$

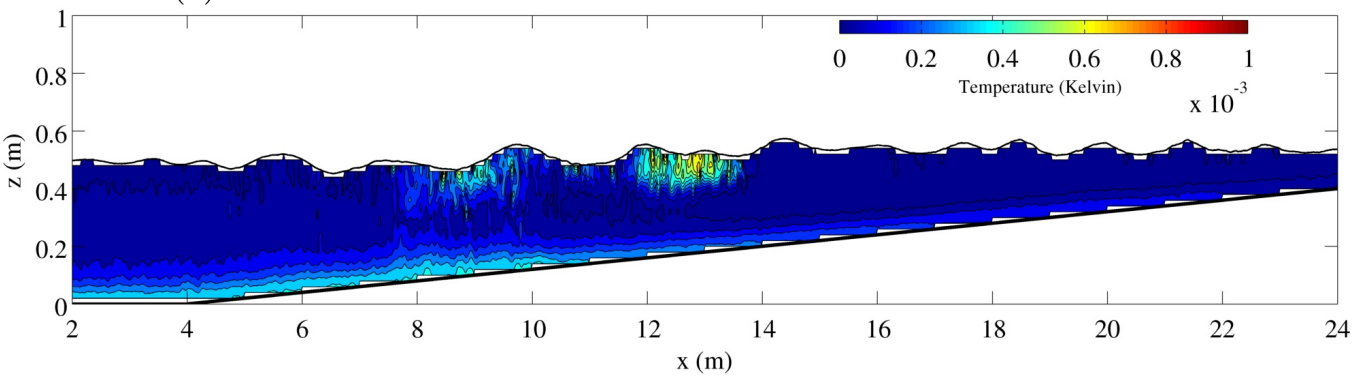

Figure 10: The cross-section view of temperature generation under short-crested wave breaking at $t=70 \mathrm{~s}$ along three sections: (a) $y=10 \mathrm{~m}$ (anti-nodal line), (b) $y=8 \mathrm{~m}$, and (c) $y=\mathbf{5} \mathbf{m}$ (nodal line or rip channel). 
- The distribution of wave heating mainly follows the pattern of wave breaking, and there is a 3D structure of internal energy generation under short-crested wave breaking.

- The magnitude of the generated internal energy or temperature by dissipation of breaking wave energy in the surf zone is relatively small. The present study found that the generated water temperature is in the order of $10^{-3}$ Kelvin for wave breaking over a typical coastal beach.

It should be mentioned that a thermally isolated system was considered in this study; but natural coast is an open environment. As a result, the generated wave heating by the dissipation of wave breaking could be quickly transferred into other forms in the open coast. We would expect that the water temperature due to the dissipation of breaking wave energy in the open coast would be smaller than the water temperature reported in this study.

\section{ACKNOWLEDGEMENTS}

The authors acknowledge the support from the Office of Naval Research, Littoral Geosciences, and Optics Program. The authors also acknowledge the ATHOS Consortium and its member organizations for their contributions to the GPUSPH code. The numerical simulations were carried out at the Maryland Advanced Research Computing Center.

\section{References}

T. Aagaard, K. P. Black, and B. Greenwood. Cross-shore suspended sediment transport in the surf zone: a field-based parameterization. Marine Geology, 185(3-4):283-302, 2002.

J. A. Battjes. Surf similarity. In Coastal Engineering 1974, pages 466-480. 1975.

D. B. Clark, S. Elgar, and B. Raubenheimer. Vorticity generation by short-crested wave breaking. Geophysical Research Letters, 39(24), 2012.

P. W. Cleary and J. Ha. Three-dimensional sph simulation of light metal components. J. Light Metals, 2(3): 169-183, 2003.

R. A. Dalrymple. A mechanism for rip current generation on an open coast. Journal of Geophysical Research, 80(24):3485-3487, 1975.

R. A. Dalrymple and B. D. Rogers. Numerical modeling of water waves with the SPH method. Coastal Engineering, 53(2):141-147, 2006.

R. G. Dean and R. A. Dalrymple. Water wave mechanics for scientists and engineers. World Scientific, Advanced Series on Ocean Engineering, 2, 1991.

D. A. Fulk. A numerical analysis of smoothed particle hydrodynamics. Technical report, Air Force Institute of Technology, Wright-Patterson AFB, OH, 1994.

R. A. Gingold and J. J. Monaghan. Smoothed particle hydrodynamics: theory and application to nonspherical stars. Monthly Notices of the Royal Astronomical Society, 181(3):375-389, 1977.

A. R. Harborne and P. J. Mumby. Novel ecosystems: altering fish assemblages in warming waters. Current Biology, 21(19):R822-R824, 2011.

A. Hérault, G. Bilotta, and R. A. Dalrymple. SPH on GPU with CUDA. Journal of Hydraulic Research, 48 (S1):74-79, 2010.

A. Hérault, G. Bilotta, A. Vicari, E. Rustico, and C. Del Negro. Numerical simulation of lava flow using a GPU SPH model. Annals of Geophysics, 54(5), 2011.

A. Iafrati. Energy dissipation mechanisms in wave breaking processes: Spilling and highly aerated plunging breaking events. Journal of Geophysical Research: Oceans, 116(C7), 2011.

N. Kumar, F. Feddersen, Y. Uchiyama, J. McWilliams, and W. OReilly. Midshelf to surfzone coupled ROMS-SWAN model data comparison of waves, currents, and temperature: Diagnosis of subtidal forcings and response. Journal of Physical Oceanography, 45(6):1464-1490, 2015. 
D. Lakehal and P. Liovic. Turbulence structure and interaction with steep breaking waves. Journal of Fluid Mechanics, 674:522-577, 2011.

L. B. Lucy. A numerical approach to the testing of the fission hypothesis. The Astronomical Journal, 82: 1013-1024, 1977.

C. V. Makris, C. D. Memos, and Y. N. Krestenitis. Numerical modeling of surf zone dynamics under weakly plunging breakers with SPH method. Ocean Modelling, 98:12-35, 2016.

J. J. Monaghan. Smoothed particle hydrodynamics. Annual Review of Astronomy and Astrophysics, 30: 543-574, 1992.

J. J. Monaghan. Simulating free surface flows with SPH. Journal of Computational Physics, 110(2):399_ 406, 1994.

J. J. Monaghan. Smoothed particle hydrodynamics. Reports on Progress in Physics, 68(8):1703, 2005.

K. Nadaoka, M. Hino, and Y. Koyano. Structure of the turbulent flow field under breaking waves in the surf zone. Journal of Fluid Mechanics, 204:359-387, 1989.

J. Pineda. Internal tidal bores in the nearshore: Warm-water fronts, seaward gravity currents and the onshore transport of neustonic larvae. Journal of Marine Research, 52(3):427-458, 1994.

R. J. Rapp and W. K. Melville. Laboratory measurements of deep-water breaking waves. Phil. Trans. R. Soc. Lond. A, 331(1622):735-800, 1990.

H. Shi, X. Yu, and R. A. Dalrymple. Development of a two-phase SPH model for sediment laden flows. Computer Physics Communications, 221(Supplement C):259 - 272, 2017.

G. Sinnett and F. Feddersen. The surf zone heat budget: The effect of wave heating. Geophysical Research Letters, 41(20):7217-7226, 2014.

E. B. Thornton. Distribution of sediment transport across the surf zone. In Coastal Engineering 1972, pages 1049-1068. 1973.

Z. Wei and R. A. Dalrymple. Numerical study on mitigating tsunami force on bridges by an SPH model. Journal of Ocean Engineering and Marine Energy, 2(3):365-380, 2016.

Z. Wei and R. A. Dalrymple. SPH modeling of vorticity generation by short-crested wave breaking. In Proceedings of 35th International Conference on Coastal Engineering, Antalya, Turkey, 2017a.

Z. Wei and R. A. Dalrymple. SPH modeling of short-crested waves. In Proceedings of 12th international SPHERIC workshop, Ourense, Spain, 2017b.

Z. Wei, R. A. Dalrymple, A. Hérault, G. Bilotta, E. Rustico, and H. Yeh. SPH modeling of dynamic impact of tsunami bore on bridge piers. Coastal Engineering, 104:26-42, 2015.

Z. Wei, R. A. Dalrymple, E. Rustico, A. Hérault, and G. Bilotta. Simulation of nearshore tsunami breaking by Smoothed Particle Hydrodynamics method. Journal of Waterway, Port, Coastal, and Ocean Engineering, 2016. doi: 10.1061/(ASCE)WW.1943-5460.0000334.

Z. Wei, R. A. Dalrymple, M. Xu, R. Garnier, and M. Derakhti. Short-crested waves in the surf zone. Journal of Geophysical Research: Oceans, 122, 2017. doi: 10.1002/2016JC012485.

Z. Wei, C. Li, R. A. Dalrymple, M. Derakhti, and J. Katz. Chaos in breaking waves. Coastal Engineering, 140:272 - 291, 2018. 